\title{
Adverse Reactions to lodinated Contrast Media
}

\author{
${ }^{1}$ Department of Internal Medicine, Virginia Commonwealth University \\ Health System, Richmond, Virginia \\ 2 Division of Cardiology, Department of Internal Medicine, Virginia \\ Commonwealth University Health System, Richmond, Virginia \\ 3 Department of Medicine/Cardiac Catheterization Laboratory, \\ McGuire Veterans Affairs Medical Center, Richmond, Virginia
}

Wendy Bottinor, $\mathrm{MD}^{1}$ Pritam Polkampally, $\mathrm{MD}^{2}$ Ion Jovin, $\mathrm{MD}^{2,3}$

\begin{abstract}
Address for correspondence Dr. Wendy Bottinor, MD, 1200 East Broad Street, 6th Floor, East Wing Room 6-202, Richmond, VA 23298,

(e-mail: wbottinor@mcvh-vcu.edu).
\end{abstract}

Int J Angiol 2013;22:149-154.

\begin{abstract}
Keywords

- iodinated contrast media

- contrast allergy

- contrast prophylaxis

- contrast nephropathy

The use of iodinated contrast agents for angiography dates back to the 1920s. With over 75 million contrast-requiring procedures performed annually worldwide, it is important to understand the risk factors, pathogenesis, diagnosis, prevention, and treatment of adverse reactions caused by iodinated contrast media (ICM). As contrast media have evolved from ionic, high-osmolality to nonionic, low-osmolality formulations the risk of reactions has decreased over time; however, no pretreatment protocol has been shown to eliminate all repeat reactions. Clinical alertness and early recognition of adverse reactions is of paramount importance and key for appropriate management of these patients. In this article, we review the most recent literature regarding adverse reactions to ICM and provide an insight into the pathogenesis, clinical presentation, pretreatment, and management of contrast-related reactions.
\end{abstract}

Iodinated contrast media (ICM) was first used in the clinical setting with the development of sodium iodide in the 1920s. The use of ICM was initially limited by poor radiographic contrast and patient toxicity. In the 1950s, technological improvements resulted in the formulation of ICM based on a tri-iodobenzoic acid ring. This formulation improved radiopacity and reduced toxicity, allowing for more widespread use of ICM. With subsequent technological advances in the 1970s, nonionic ICM and dimeric ICM were developed. Today, four categories of ICM exist: ionic monomeric, nonionic monomeric, ionic dimeric, and nonionic dimeric. These categories differ in three significant ways: iodine atom-to-particle ratio, osmolality, and viscosity. ${ }^{1,2}$

Ionic monomeric ICM has the lowest iodine-to-particle ratio of $1.5: 1$; it also has the highest osmolality ranging from five to eight times that of blood. Nonionic monomeric and ionic dimeric ICM have the same iodine-to-particle ratio of $3: 1$. The osmolality of these preparations are approximately twice that of blood. Nonionic dimeric contrast has the highest iodine-to-particle ratio of $6: 1$. It also has the most physiologic osmolality, approximately equal to that of blood. In general, as osmolality decreases, viscosity increases. This increase in viscosity can prove problematic in applications requiring high flow rates of ICM. ${ }^{3}$

As the technology of ICM has broadened, so too has the wide array of applications for this technology. ICM is now used in over 75 million procedures worldwide each year. ${ }^{4}$ It has proven useful in the imaging of a variety of anatomic structures including solid organs, vasculature, free spaces such as the abdominal cavity, and extremities. It is useful for different imaging modalities, including both X-ray and magnetic resonance imaging. In the 1950s, with the expanded use of ICM, reports of adverse reactions, including death, began to surface. These reports prompted the first large, multicenter, prospective study in 1975, which estimated the incidence of contrast reaction at approximately $5 \%{ }^{5}$ Recent estimates of all adverse reactions to ICM range from 1 to $12 \%$, with severe reactions comprising only 0.01 to $0.2 \%$ of total reactions. ${ }^{6,7}$

The risk of reactions has decreased over time as contrast media have evolved from ionic, high-osmolality to nonionic, low-osmolality formulations; however, the expense and viscosity of the low-osmolality agents limit their universal use.

In addition, the ability of low-osmolality agents to reduce the incidence of severe life-threatening reactions is still published online

August 16, 2013
Copyright @ 2013 by Thieme Medical Publishers, Inc., 333 Seventh Avenue, New York, NY 10001, USA. Tel: +1(212) 584-4662. 
debated. Although Katayama et al observed a decrease in severe adverse reactions when low-osmolality agents were used, $^{6}$ further research by Lasser et al has noted higher incidences of severe reactions in patients exposed to lowosmolality agents. ${ }^{8}$ One proposed explanation for this difference is selective of high-risk patients to receive low osmolality.

\section{Classification}

Adverse contrast reactions are typically divided into two categories: immediate reactions and delayed reactions. Immediate reactions, which occur less than 1 hour after contrast administration, were initially postulated to be largely anaphylactoid rather than true immunoglobulin E (IgE)-mediated allergic reactions. These reactions were thought to be generated by the activation of complement, fibrinolytic systems, kinin systems, and the release of histamine, prostaglandins, bradykinins, and other mediators. ${ }^{9}$ However, more recently, evidence of histamine and tryptase release, as well as positive skin testing, has suggested a role for IgE-mediated allergy even in the development of immediate reactions. ${ }^{10,11}$

Delayed reactions occur 1 hour to 7 days after contrast administration. Current evidence including typical time course, positive patch tests, delayed intradermal tests, and increased risk of reaction in patients treated with interleukin2 or with active systemic lupus erythematosus all point to a Tcell-mediated pathogenesis. ${ }^{12-14}$

The exact mechanism of this T-cell activation is unknown, but direct binding to the major histocompatibility complexT-cell receptor complex or binding after processing by antigen-presenting cells has recently been proposed..$^{15}$

The most common clinical manifestations of adverse reactions are dermatologic. Pruritus and urticaria occur in approximately $70 \%$ of adverse reactions. ${ }^{6}$

Flushing has also been observed in the acute setting. An even wider variety of delayed manifestations exist including exanthema, erythema multiforme, vasculitis, Stevens-Johnson syndrome, toxic epidermal necrolysis, and graft versus host reaction. ${ }^{1,13}$

Cardiac manifestations have also been known to occur. Immediate cardiac findings include cardiovascular shock, cardiac arrhythmia, and cardiac arrest. Debate currently exists as to whether these cardiac manifestations are a secondary result of homeostatic dysfunction (i.e., vasodilation), reduced venous return and volume loss, or due to a more direct cardiac mechanism (i.e., Kounis syndrome). ${ }^{16,17}$

Factors arguing in favor of Kounis syndrome, or cardiac dysfunction secondary to the direct cardiac effect of inflammatory mediators, include observed heterogeneity in degranulation of mast cells from different human tissues as well as increased serum IgE levels in patients with myocardial infarction or unstable angina. ${ }^{18}$ It is postulated that degranulation of cardiac mast cells may be the direct cause of cardiac manifestations. Although cardiac manifestations are less common in delayed reactions, hypotension and cardiovascular shock have been reported in isolated cases in which nonionic dimeric ICM were used. ${ }^{19,20}$
Systemic manifestations of adverse reactions have also been reported. The severity of these reactions can vary greatly. Headache, nausea, vomiting, and arm pain can occur in mild reactions. These symptoms are more common with the use of high-osmolality agents. ${ }^{3}$ Intermediate reactions can result in hypotension and bronchospasm. Patients experiencing severe reactions can develop respiratory arrest, angioedema, convulsions, and loss of consciousness. ${ }^{1,13}$ As mentioned above, these reactions occur in $0.2 \%$ or less of individuals. Systemic manifestations, although rare, are most consistently observed with immediate adverse reactions.

Clinical observation and laboratory work have helped to elucidate several pathogenic possibilities that can aid in classification of immediate adverse reactions. Accordingly, acute symptoms can be subcategorized as vasomotor, vagal type, or anaphylactoid. Vasomotor responses present with transient warmth, nausea, and emesis; vagal reactions manifest as bradycardia-associated hypotension. Anaphylactoid reactions range from minor to severe and occur within 30 minutes of contrast administration. They appear clinically identical to anaphylaxis but lack an IgE-dependent mechanism. Minor anaphylactoid reactions include transient nausea, vomiting, urticaria, pruritus, and diaphoresis. Moderate reactions present with faintness, prolonged vomiting and urticaria, facial and laryngeal edema, and mild bronchospasm. ${ }^{21}$ The hallmarks of a severe reaction include hypotensive shock, pulmonary edema, respiratory or cardiac arrest, and convulsions. ${ }^{22}$

Predisposing factors for adverse contrast reactions have also been identified. The most significant risk factor is a previous reaction to ICM. Individuals with a previous history of ICM reaction have a 2.5 to $44 \%$ risk of reaction upon reexposure. Those with a history of asthma have a 10 times higher risk of a severe reaction. In addition, any history of allergy increases the risk of reaction by a factor of 3. Cardiac disease, dehydration, hematologic conditions, metabolic conditions, and extremes of age have also been implicated as risk factors. ${ }^{1,6,23,24}$

Historically, shellfish and sea food allergy have been linked to ICM. This is likely based on observations by Shehadi indicating that several types of food allergy, in particular shellfish and sea food, increase the risk for an adverse reaction to ICM. ${ }^{5}$ Underlying pathophysiology supporting this observation is unclear. Both fish and shellfish contain iodine, but iodine is not and cannot be an allergen. Iodine is found throughout our bodies in thyroid hormones and amino acids. The major allergens in shellfish are tropomyosins, which are proteins important in muscle contraction and which have no relation to iodine. Current guidelines do not suggest treatment plan modification, specifically prophylactic pretreatment, based on a history of shellfish or seafood allergy alone. $^{25}$

Contrast-induced acute kidney injury or nephropathy (CIN) is a significant adverse complication of ICM which is linked with excess morbidity and mortality. ${ }^{26,27}$ Chronic kidney disease is perhaps the most well-known risk factor for CIN. Additional risk factors have been identified. Diabetes mellitus, periprocedural hypotension, dehydration or volume 
contraction, age $>70$ years, congestive heart failure, myocardial infarction within the previous 24 hours, and multiple doses of contrast media are all associated with increased risk of $\mathrm{CIN}^{28}$

With the use of nonionic, dimeric contrast media, concerns regarding an increase in thrombogenicity have arisen. ${ }^{29}$ However, more recent studies have shown no increase, and in fact, a decrease in thrombogenicity with these agents at the higher concentrations expected during injection. The underlying mechanism is thought to be inhibition of platelet aggregation secondary to impaired intracellular calcium release. ${ }^{30,31}$

\section{Prophylaxis and Treatment}

Fluid volume loading is the single most important measure that can be taken before intravascular CM administration. Sodium chloride is commonly used in this application; however, some have advocated for the use of sodium bicarbonate. A recent meta-analysis did indicate some reduction in CIN with the use of sodium bicarbonate; however, this has not seemed to translate into clinically relevant end points such as decreased mortality or reduced hemodialysis. ${ }^{28,32}$

Although steroid prophylaxis is not recommended for all patients, given our ability to identify those individuals most likely to suffer an adverse ICM reaction, some have argued for prophylactic therapy for high-risk individuals. ${ }^{22,23}$ The most recent guidelines from two independent societies has advocated the use of premedication in patients with history of previous ICM reaction. The European Society of Urogenital Radiology extends this recommendation to include patients with a history of asthma or allergy requiring medical treatment. No definitive guidelines are provided for those patients receiving nonionic contrast, likely given the inherent lower rate of allergic reaction to nonionic media. ${ }^{25,33}$ Several different prophylactic regimens have been introduced. Corticosteroids are the corner stone of these regimens with $\mathrm{H} 1 / \mathrm{H} 2$ blockers occasionally recommended in conjunction. One of the commonly followed prophylactic regimens involves $50 \mathrm{mg}$ of oral prednisone given 13,7 , and 1 hours before ICM administration and additionally $50 \mathrm{mg}$ of oral diphenhydramine 1 hour before ICM. Evidence for this algorithm is based on findings by Greenberger and Patterson showing the risk of an immediate adverse reaction in patients with previous history of adverse reaction decreased from 9.1 to $0.5 \%$ when nonionic monomeric media and the prophylactic regimen above were used. ${ }^{34}$

Lasser et al have also demonstrated a reduction in the incidence of reactions from 4.9 to $1.7 \%$ when $32 \mathrm{mg}$ of oral methylprednisolone was given 6 to 24 hours before contrast administration and again 2 hours before administration. ${ }^{35}$

Prophylactic regimens have been documented to reduce the frequency of adverse reactions. No pretreatment protocol, however, has been shown to eliminate all repeat reactions.

Table 1 Emergent treatment of adverse reactions to iodinated contrast media

\begin{tabular}{|c|c|}
\hline Reaction & Treatment \\
\hline \multirow[t]{6}{*}{ Severe bronchospasm } & High flow supplemental oxygen \\
\hline & Inhaled $\beta_{2}$ agonist \\
\hline & Epinephrine injection \\
\hline & Diphenhydramine IV \\
\hline & Hydrocortisone IV \\
\hline & $\mathrm{H}_{2}$ blocker IV \\
\hline \multirow[t]{6}{*}{ Facial and laryngeal edema } & Consider intubation in setting of stridor or airway compromise \\
\hline & High flow supplemental oxygen \\
\hline & Epinephrine injection \\
\hline & Diphenhydramine IV \\
\hline & Hydrocortisone IV \\
\hline & $\mathrm{H}_{2}$ blocker IV \\
\hline \multirow[t]{4}{*}{ Hypotension with bradycardia } & Elevate patient's lower extremities \\
\hline & High flow supplemental oxygen \\
\hline & Rapid IV fluid resuscitation \\
\hline & Epinephrine injection \\
\hline \multirow[t]{4}{*}{ Hypotension and tachycardia } & Elevate patient's lower extremities \\
\hline & High flow supplemental oxygen \\
\hline & Rapid IV fluid resuscitation \\
\hline & Epinephrine injection \\
\hline
\end{tabular}


Thus clinical alertness and early recognition of adverse reactions is of paramount importance and key for appropriate management of these patients. ${ }^{36}$

Once an adverse reaction to ICM has occurred, treatment is based on symptomatology and underlying pathophysiology. Mild reactions such as pruritus and urticaria are usually selflimiting and do not require treatment. IV access should be maintained and the individual should be observed to ensure that severe symptoms do not develop. Mild-to-moderate bronchospasm can be treated with supplemental oxygen and an inhaled $\beta_{2}$ agonist. More severe reactions may require the administration of epinephrine and aggressive volume resuscitation. Individuals trained in cardiopulmonary resuscitation and emergency drugs and supplies should always be readily available. ${ }^{1}$ A symptom-specific treatment algorithm is provided in - Table $\mathbf{1}$.

\section{Conclusions}

The development of nonionic low-osmolar ICM has greatly reduced the incidence of adverse reactions to contrast media, however, adverse reactions still occur. The majority of these reactions are self-limited and predominately involve dermatologic manifestations. Premedication with steroids is only recommended for individuals identified as high risk of an adverse reaction to ICM. Life-threatening reactions occur in up to $0.2 \%$ of individuals depending on the type of ICM used. Physicians performing diagnostic or therapeutic procedures using contrast dye must be aware of the potential for these reactions and be prepared to efficiently treat patients suffering from severe adverse reactions.

\section{References}

1 Morcos SK, Thomsen HS. Adverse reactions to iodinated contrast media. Eur Radiol 2001;11(7):1267-1275

2 Singh J, Daftary A. Iodinated contrast media and their adverse reactions. J Nucl Med Technol 2008;36(2):69-74, quiz 76-77

3 Thomsen HS, Morcos SK. Radiographic contrast media. BJU Int 2000;86(Suppl 1):1-10

4 Christiansen C. X-ray contrast media-an overview. Toxicology 2005;209(2):185-187

5 Shehadi $\mathrm{WH}$. Adverse reactions to intravascularly administered contrast media. A comprehensive study based on a prospective survey. Am J Roentgenol Radium Ther Nucl Med 1975;124(1): 145-152

6 Katayama H, Yamaguchi K, Kozuka T, Takashima T, Seez P, Matsuura $\mathrm{K}$. Adverse reactions to ionic and nonionic contrast media. A report from the Japanese Committee on the Safety of Contrast Media. Radiology 1990;175(3):621-628

7 Bush WH, Swanson DP. Acute reactions to intravascular contrast media: types, risk factors, recognition, and specific treatment. AJR Am J Roentgenol 1991;157(6):1153-1161

8 Lasser EC, Lyon SG, Berry CC. Reports on contrast media reactions: analysis of data from reports to the U.S. Food and Drug Administration. Radiology 1997;203(3):605-610

9 Almén T. The etiology of contrast medium reactions. Invest Radiol 1994;29(Suppl 1):S37-S45

10 Laroche D, Namour F, Lefrançois C, et al. Anaphylactoid and anaphylactic reactions to iodinated contrast material. Allergy 1999;54(Suppl 58):13-16
11 Dewachter P, Laroche D, Mouton-Faivre C, et al. Immediate reactions following iodinated contrast media injection: a study of 38 cases. Eur J Radiol 2011;77(3):495-501

12 Christiansen $C$. Late-onset allergy-like reactions to X-ray contrast media. Curr Opin Allergy Clin Immunol 2002;2(4):333339

13 Brockow K, Christiansen C, Kanny G, et al; ENDA; EAACI interest group on drug hypersensitivity. Management of hypersensitivity reactions to iodinated contrast media. Allergy 2005;60(2): 150-158

14 Lerch M, Keller M, Britschgi M, et al. Cross-reactivity patterns of T cells specific for iodinated contrast media. J Allergy Clin Immunol 2007;119(6):1529-1536

15 Keller M, Lerch M, Britschgi M, et al. Processing-dependent and -independent pathways for recognition of iodinated contrast media by specific human T cells. Clin Exp Allergy 2010;40(2): 257-268

16 Kounis NG, Davlouros P, Hahalis G, Mazarakis A. The heart seems to be the primary site and the target of anaphylaxis resulting in the development of Kounis syndrome. Intern Emerg Med 2012;7 (Suppl 2):S119-S120

17 Matucci A, Vultaggio A, Fassio F, Rossi O, Maggi E. Heart as the early main target of severe anaphylactic reactions: two case reports. Intern Emerg Med 2011;6(5):467-469

18 Patella V, Marinò I, Lampärter B, Arbustini E, Adt M, Marone G. Human heart mast cells. Isolation, purification, ultrastructure, and immunologic characterization. J Immunol 1995;154(6):28552865

19 Yoshikawa H. Late adverse reactions to nonionic contrast media. Radiology 1992;183(3):737-740

20 Burton PR, Jarmolowski E, Raineri F, Buist MD, Wriedt CH. A severe, late reaction to radiological contrast media mimicking a sepsis syndrome. Australas Radiol 1999;43(3):360-362

21 Canter LM. Anaphylactoid reactions to radiocontrast media. Allergy Asthma Proc 2005;26(3):199-203

22 Hagan JB. Anaphylactoid and adverse reactions to radiocontrast agents. Immunol Allergy Clin North Am 2004;24(3):507-519, vii-viii

23 Wolf GL, Mishkin MM, Roux SG, et al. Comparison of the rates of adverse drug reactions. Ionic contrast agents, ionic agents combined with steroids, and nonionic agents. Invest Radiol 1991;26 (5):404-410

24 Thomsen HS, Bush WH Jr. Adverse effects of contrast media: incidence, prevention and management. Drug Saf 1998;19(4): 313-324

25 Levine GN, Bates ER, Blankenship JC, et al. 2011 ACCF/AHA/SCAI Guideline for Percutaneous Coronary Intervention: a report of the American College of Cardiology Foundation/American Heart Association Task Force on Practice Guidelines and the Society for Cardiovascular Angiography and Interventions. Circulation 2011; 124(23):e574-e651

26 Rihal CS, Textor SC, Grill DE, et al. Incidence and prognostic importance of acute renal failure after percutaneous coronary intervention. Circulation 2002;105(19):2259-2264

27 From AM, Bartholmai BJ, Williams AW, Cha SS, McDonald FS Mortality associated with nephropathy after radiographic contrast exposure. Mayo Clin Proc 2008;83(10):1095-1100

28 Stacul F, van der Molen AJ, Reimer P, et al; Contrast Media Safety Committee of European Society of Urogenital Radiology (ESUR). Contrast induced nephropathy: updated ESUR Contrast Media Safety Committee guidelines. Eur Radiol 2011;21(12):25272541

29 Esplugas E, Cequier A, Jara F, et al. Risk of thrombosis during coronary angioplasty with low osmolality contrast media. Am J Cardiol 1991;68(10):1020-1024

30 Schräder R. Thrombogenic potential of non-ionic contrast mediafact or fiction? Eur J Radiol 1996;23(Suppl 1):S10-S13 
31 Georgakis A, Ener RA, Jin J, et al. Risk of thrombogenicity among nonionic radiocontrast agents. J Invasive Cardiol 2008;20(7):349-353

32 Trivedi H, Nadella R, Szabo A. Hydration with sodium bicarbonate for the prevention of contrast-induced nephropathy: a metaanalysis of randomized controlled trials. Clin Nephrol 2010;74 (4):288-296

33 Morcos SK, Thomsen HS, Webb JA; Contrast Media Safety Committee of the European Society of Urogenital Radiology. Prevention of generalized reactions to contrast media: a consensus report and guidelines. Eur Radiol 2001;11(9):1720-1728
34 Greenberger PA, Patterson R. The prevention of immediate generalized reactions to radiocontrast media in high-risk patients. J Allergy Clin Immunol 1991;87(4):867-872

35 Lasser EC, Berry CC, Mishkin MM, Williamson B, Zheutlin N, Silverman JM. Pretreatment with corticosteroids to prevent adverse reactions to nonionic contrast media. AJR Am J Roentgenol 1994;162(3):523-526

36 Williams AN, Kelso JM. Radiocontrast-induced anaphylaxis despite pretreatment and use of iso-osmolar contrast. Ann Allergy Asthma Immunol 2007;99(5):467-468 